\title{
Redefining priorities in gene-based drug discovery
}

\author{
Mehran M. Khodadoust and Thomas P. Klein
}

The belief that genomics will rapidly and directly lead to a host of new drug products has caused a number of major pharmaceutical and biotechnology players to establish as a priority the patenting of as many genes and gene targets as possible. Yet, many of those companies that were swift to jump on the genomics bandwagon are now learning there is no fast route from gene to blockbuster pharmaceutical. What's more, these burgeoning intellectual property portfolios may well become obsolete as systems-based approaches to drug discovery become widely adopted.

The incredible level of functional complexity revealed as we decode the genome should come as no surprise. New molecular tools only confirm what we had already guessed-all life on Earth is part of complex systems, from the biosphere, to social networks, down to the cellular and molecular levels. Huang ${ }^{1}$ points out that in complex organisms, each gene product is part of a network or pathway that, in many cases, may represent only a single step in a series of complex intracellular or extracellular interactions. As Huang says: "The 'function' of a gene product appears to depend on its 'cellular context?"' Thus, function derives from an architecture that cannot be determined by examining the building blocks alone.

At its simplest, the genomics endeavor has only given us a list of biological components. Only by understanding the functions of gene products within the cell and tying them to biological processes can we then determine what is a candidate for pharmacological manipulation-a so-called druggable target. Establishing the pharmaceutical value of any individual gene entails the discovery and validation of its role in a disease process. Ideally, we need to elucidate its unique role in a disease-associated regulatory pathway. However, Thanos and Maniatis ${ }^{2}$ (and others) have pointed out that genetic regulatory mechanisms at the cellular level are highly complex, reflecting the intricate nature of signaling pathways. This is further complicated by issues of cell-type specificity. Functions in one cell type-or even one species_-may differ in another.

A central problem is whether the relationships between a gene, its clinical applicability,

Mehran Khodadoust is president and Thomas Klein is executive vice president of Bionaut

Pharmaceuticals, Boston, MA

(MKhodadoust@BionautPharma.com). and the resulting product are linear. "Linear" refers to the concept that biological regulatory systems consist of a series of unique causeand-effect relationships. There are instances in which such linearity exists: some highly successful biopharmaceutical products, including insulin, erythropoietin, and granulocyte colony stimulating factor, operate through their ability to modulate such relationships.

However, unique cause-and-effect relationships are the exception and not the rule. Developing receptor ligands as therapeutics is complicated by ligand redundancies and cell-type specificity. Classes of ligands exhibiting these characteristics include vascular endothelial growth factors (VEGFs), fibroblast growth factors (FGFs), interleukins (IL-10, IL-12, IL-18, etc.), transforming growth factor- $\beta$ (TGF- $\beta$ ), and tumor necrosis factors (TNFs). Redundancy and cell-type specificity issues have been behind the failure of a number of these protein therapeutics in clinical trials. The nature of the pharmacological effects of these ligands is further complicated by nonlinear dose dependencies. $\mathrm{Ma}^{3}$ describes this as a "double-edged sword": TNF- $\alpha$ and IL-12, for example, may exhibit either pro- or antiinflammatory effects, depending on the dosage. Similar effects have also been observed with TGF- $\beta$, which also demonstrates issues of ligand redundancy and celltype specificity 4 .

Today, proteomics is in vogue in industry and the media. Unfortunately, it risks disappointing the pharmaceutical industry for many of the same reasons as genomics-by ignoring underlying biological complexities. The scientific priority today should not be a headlong effort to develop more raw data, but rather to develop new and powerful tools to address the challenges posed by cellular regulatory complexities and to more efficiently use the data at hand.

There are two alternative approaches for addressing signaling pathway complexities. The first, and arguably the more direct, is to face the problem straight on and to attempt to unravel and understand biological networks. Techniques that may be useful for the identification of individual pathway components include proteomics, multi-hybrid systems, expression cloning, and DNA chips. These methods work either by inferring function from direct or indirect association between individual signaling components or by hypothesizing function from correlation of events. However, such methods are laborious, time-consuming, and often deliver ambiguous results. The hunt for alternatives to insulin offers a clear example of the weakness of this approach: Over two decades of work by an estimated 75 different companies has failed to produce an oral antidiabetic as effective as injectable insulin. Key obstacles in this search have been the presence of cell-typespecific and overlapping regulatory pathways, including unforeseen liver toxicities. So, how directly does this "means" lead to an "end"?

An alternative route is to start with the "end". For example, engineered, cell-based assays can be used to screen drug candidates for efficacy directly against specific diseaserelated genetic sites using high-throughput techniques. Such screens are based on the activity of reporter genes introduced into cells that directly indicate the activity of a gene at any regulated site within the genome.

For example, a reporter gene can be delivered into every genetic site regulated by insulin. Each cell clone isolated using this method can then be used either to monitor activity of insulin or screen for new drug candidates-whether they stimulate or inhibit targeted insulin pathway(s). In a sense, this is a molecular version of the traditional pharmaceutical approach - to create a disease model and then screen drugs to see what works. The isolation and use of multiple cell lines, each embodying a unique reporter gene, allows early and rapid screening for pathway redundancy, specificity, and adverse side-effects-issues responsible for the substantial portion of drug failures during downstream clinical trials.

Furthermore, using such assays, new chemical entities can be identified and validated without ever screening against any specific, potentially patented, gene target. Clearly, such techniques have the power to bypass substantial portions of the gene-based intellectual property rights in which so many firms have invested so much. Ultimately, the priority must be to focus on the most direct path to drug identification and development. That means focusing on cellular regulatory complexity rather than amassing stockpiles of poorly characterized intellectual property.

1. Huang, S. Nat. Biotechnol. 18, 471-472 (2000)

2. Thanos, D. and Maniatis, T. Cell 83, 1091-1100 (1995).

3. Ma, X., Microbes Infect. 3, 121-129 (2001).

4. Omer, F.M., Kurtzhals, J.A.L., and Riley, E.M. Parasitol. Today 16, 18-23 (2000). 